\title{
Inflammatory myofibroblastic tumors arising from pancreas head and peri-splenic area mimicking a malignancy
}

\author{
Eun Jeong Jang ${ }^{1}$, Kwan Woo Kim¹, Sung Hwa Kang ${ }^{1}$, Min Gyoung $\mathrm{Pak}^{2}$, and Song Hee Han² \\ Departments of ${ }^{1}$ Surgery and ${ }^{2}$ Pathology, Dong-A University Hospital, \\ Dong-A University College of Medicine, Busan, Korea
}

\begin{abstract}
Inflammatory myofibroblastic tumors (IMTs) are a rare chronic inflammatory disease with unclear pathogenesis and pathological features that are not those of a malignant tumor. It is difficult to differentially diagnose them without surgical excision because of their unpredictable clinical behavior, which ranges from benign to locally invasive aggressiveness. We report two cases of IMTs that were diagnosed after surgery. In one case, the IMT originated in peri-splenic area in a 63-year-old female patient. The other case involved a 48-year-old female patient who suffered from an IMT of the head of the pancreas. Both of these cases did not require further treatment based on histological findings, and there has been no evidence of recurrence or metastasis so far. These cases show that the primary choice for the exact diagnosis and proper treatment of IMTs is complete surgical resection. (Ann Hepatobiliary Pancreat Surg $2021 ; 25: 287-292$ )
\end{abstract}

Key Words: Inflammatory myofibroblastic tumor; Pancreas; Retroperitoneum; Case report

\section{INTRODUCTION}

Inflammatory myofibroblastic tumors (IMTs) have been reported to occur in multiple anatomical locations, including the lungs, bladder, spleen, breast, pancreas, liver, colon, spermatic code, prostate, and soft tissue. ${ }^{1}$ An IMT is a fibroblastic neoplasm composed of spindle cells accompanied by an inflammatory infiltrate of plasma cells, lymphocytes, or eosinophils. ${ }^{2}$ The incidence of IMTs is approximately $0.04-0.7 \%$, and clinical data have shown that they have a $25 \%$ rate of local recurrence and up to a $5 \%$ rate of distant metastasis. ${ }^{3}$

The symptoms of IMT are ambiguous and generally related to obstructive symptoms caused by the large-sized mass. $^{2}$ Additionally, when a mass is found in the pancreas, IMTs are most commonly mistaken for pancreatic cancer according to their clinical and radiological characteristics. Therefore, the initial diagnosis of IMTs is difficult without extensive histological examination. Usually, they are diagnosed by histological evaluations after surgery.
In this study, we report two cases of IMTs that were diagnosed after surgery. The first case arose in peri-splenic area of a 63-year-old female patient who underwent complete mass excision. The second case occurred in a 48-year-old female patient who underwent pylorus-preserving pancreaticoduodenectomy (PPPD) due to a pancreas-head mass.

\section{CASE}

\section{Case 1}

A 63-year-old female patient with diabetes mellitus and hypertension had been suffering from fever and sweating, which were controlled easily for several months. After visiting a local clinic, she was referred to our hospital for the evaluation of an abdominal mass $>10 \mathrm{~cm}$ in size, which was strongly suspicious for lymphoma, leiomyosarcoma, or malignant fibrous histiocytoma in the left upper quadrant. Laboratory findings showed a slight increase in the white blood cell count (14.93; normal range: 3-9.3

Received: January 10, 2021; Revised: March 4, 2021; Accepted: March 4, 2021

Corresponding author: Kwan Woo Kim

Department of Surgery, Dong-A University College of Medicine, 26 Daesingongwon-ro, Seo-gu, Busan 49201, Korea Tel: +82-51-240-5146, Fax: +82-51-247-9316, E-mail: d002045@naver.com

Copyright (C) 2021 by The Korean Association of Hepato-Biliary-Pancreatic Surgery

This is an Open Access article distributed under the terms of the Creative Commons Attribution Non-Commercial License (http://creativecommons.org/ licenses/by-nc/4.0) which permits unrestricted non-commercial use, distribution, and reproduction in any medium, provided the original work is properly cited. Annals of Hepato-Biliary-Pancreatic Surgery • pISSN: 2508-5778 - elSSN: 2508-5859 
$\left.10^{3} / \mathrm{ul}\right)$ and C-reactive protein (CRP) (1.47; normal range: 0-0.5 mg/dl). However, no particular abnormality was noticed. Moreover, a tumor marker test showed normal results.

Enhanced abdominal computed tomography (CT) showed a $10-\mathrm{cm}$ heterogeneous mass extending in the peri-splenic area (Fig. 1). No metastatic lesions were observed in the enhanced chest CT and abdominal CT scans. We did not perform any pre-operative biopsies due to the patient's refusal and the clinical judgment that complete surgical excision was necessary for a definite pathologic diagnosis. The patient underwent radical excision of the retroperitoneal mass. During the operation, a huge mass was found that strongly adhered to the posterior portion of the spleen, upper pole of the left kidney, and the diaphragm. Therefore, diaphragmatic excision was performed simultaneously. The patient was discharged on the 9th day after surgery without any peri- or post-operative complications.

Upon histological examination, the mass was diagnosed as an IMT associated with frequent IgG4-positive cell infiltration. Grossly, it was a grayish, encapsulated mass with dimensions of $13 \mathrm{~cm} \times 9.5 \mathrm{~cm}$. Immunochemical studies showed negative results for C-kit, S100, and P53, along with positive results for anaplastic lymphoma kinase (ALK), smooth muscle actin (SMA), vimentin, desmin, CD68, and phosphorylated histone $\mathrm{H} 3$ (PHH3) (Fig. 2). As of two years after surgery, the patient has had no local recurrence or metastasis without further treatment, which was determined through multidisciplinary opinions. Fig. 3 shows the CT findings from the latest follow-up.

\section{Case 2}

A 48-year-old female patient was referred to our hospital after regular health examinations revealed the presence of hyperbilirubinemia and an abdominal mass. She had a medical history of hypertension. The initial laboratory findings were as follows: alanine transaminase (AST), 178 (normal range: 0-35 U/L); aspartate transaminase (ALT), 77 (normal range: 0-35 U/L); alkaline phosphatase (ALP), 1,295 (normal range: 30-120 U/L); total bilirubin, 3.9 (normal range: $0.2-1.2 \mathrm{mg} / \mathrm{dl}$ ); direct bilirubin, 2.2 (normal range: 0-0.4 mg/dl), alpha-fetoprotein (AFP), 6.58 (normal range:
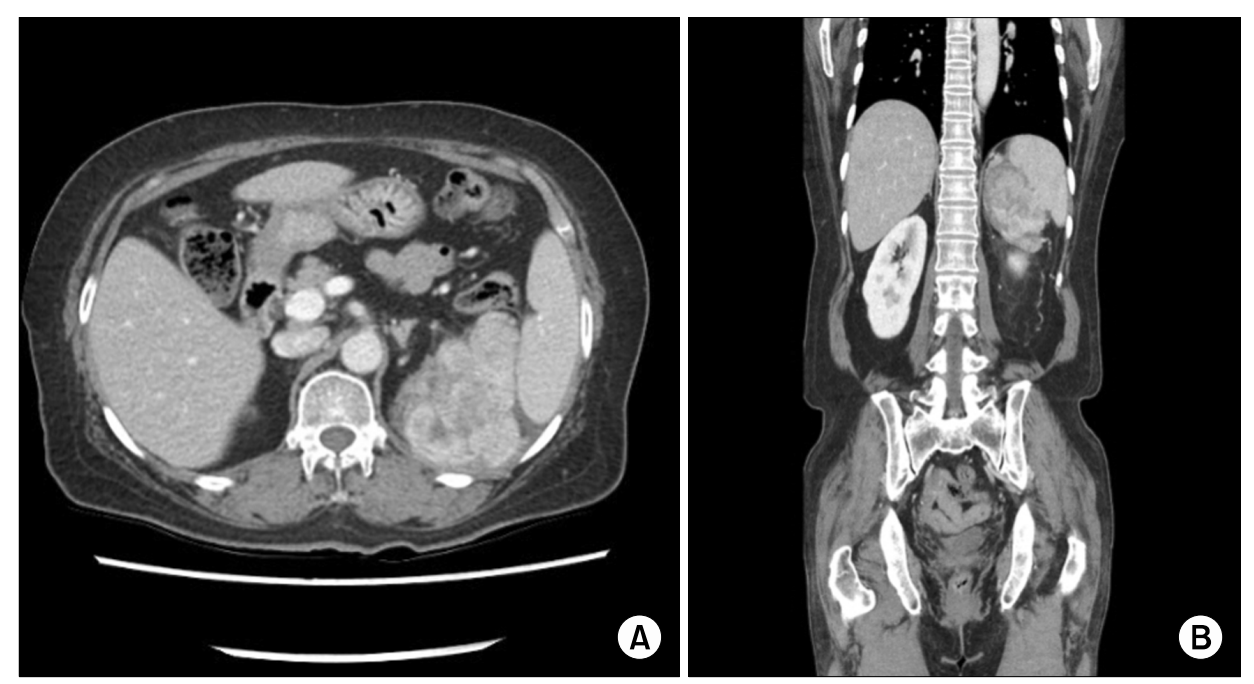

Fig. 1. (A, B) Abdominal CT shows $10 \mathrm{~cm}$ heterogenous mass.
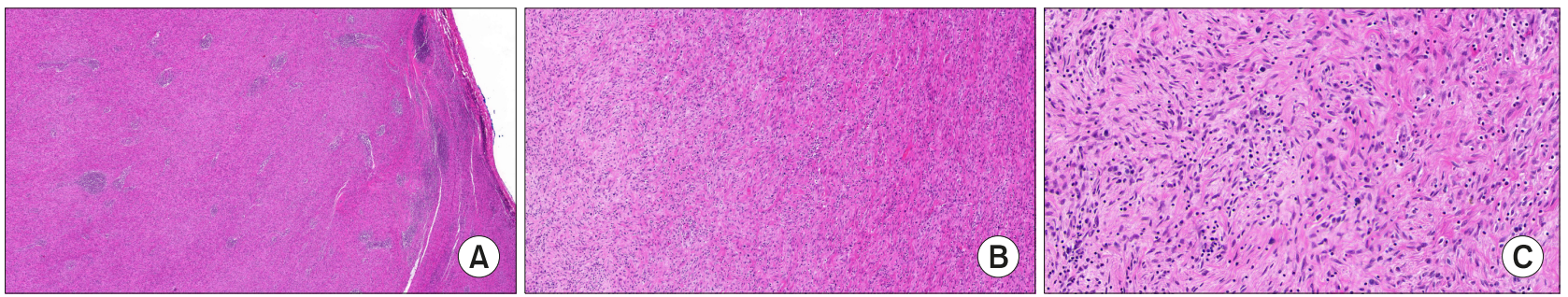

Fig. 2. The histopathological evaluation shows a well-circumscribed whitish mass characterized by the proliferation of spindle cells mixed with inflammatory cells such as lymphocytes and plasma cells, compatible with Inflammatory myofibroblastic tumor. $\times 10$ magnification $(\mathrm{A}), \times 100$ magnification $(\mathrm{B}), \times 400$ magnification $(\mathrm{C})$. 
0-7 ng/ml); carcinoembryonic antigen (CEA), 2.34 (normal range: 0-5 ng/ml); carbohydrate antigen 19-9 (CA19-9), 235 (normal range: 0-27 U/ml).

$\mathrm{CT}$ and magnetic resonance imaging (MRI) findings re-

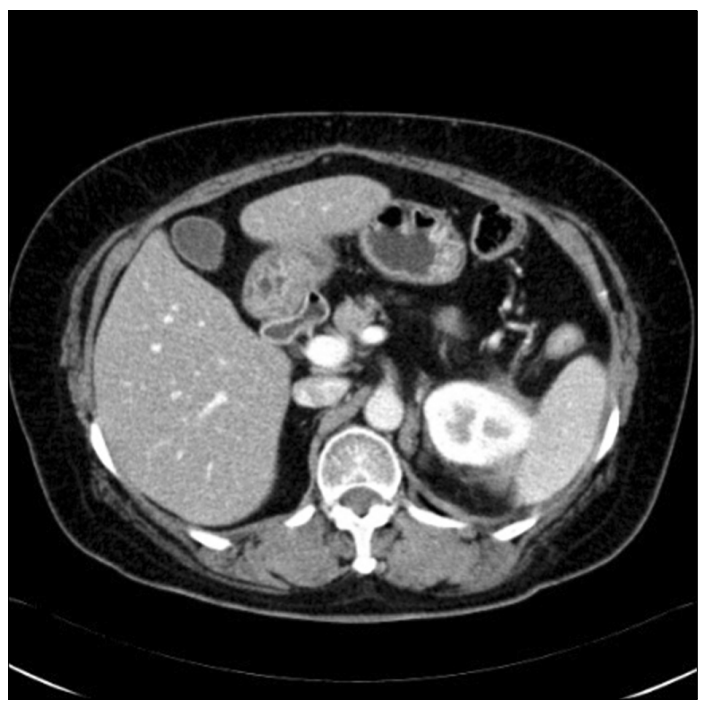

Fig. 3. Two years after surgery, CT shows no local recurrence and metastasis. vealed a $7.5-\mathrm{cm}$ mass with obstructive jaundice in the head of the pancreas, which was suspected to be either a solid pseudopapillary tumor or retroperitoneal solitary fibrous tumor (Fig. 4A-C). Moreover, a huge hypermetabolic mass was observed in the head of the pancreas without any metastatic lesions using positron emission tomography (PET) (Fig. 4D). The patient underwent pylorus-preserving pancreaticoduodenectomy (PPPD) for accurate pathologic diagnosis and complete surgical excision without pre-operative biopsy. During the operation, there was no evidence of distal organ or lymph node metastasis. The patient was discharged routinely after 12 days without any surgical or medical complications.

After the operation, the final pathological diagnosis was IMT. The tumor was relatively well defined with dimensions of $8.9 \mathrm{~cm} \times 5.7 \mathrm{~cm}$ and involving the duodenal wall. A total of 23 lymph nodes were retrieved during surgery, but all were negative. The tumor was composed of fascicles of plump spindle cells with oval nuclei and small nucleoli (Fig. 5A). There was no necrosis or hemorrhage. Inflammatory cells (mostly plasma cells) were either seen
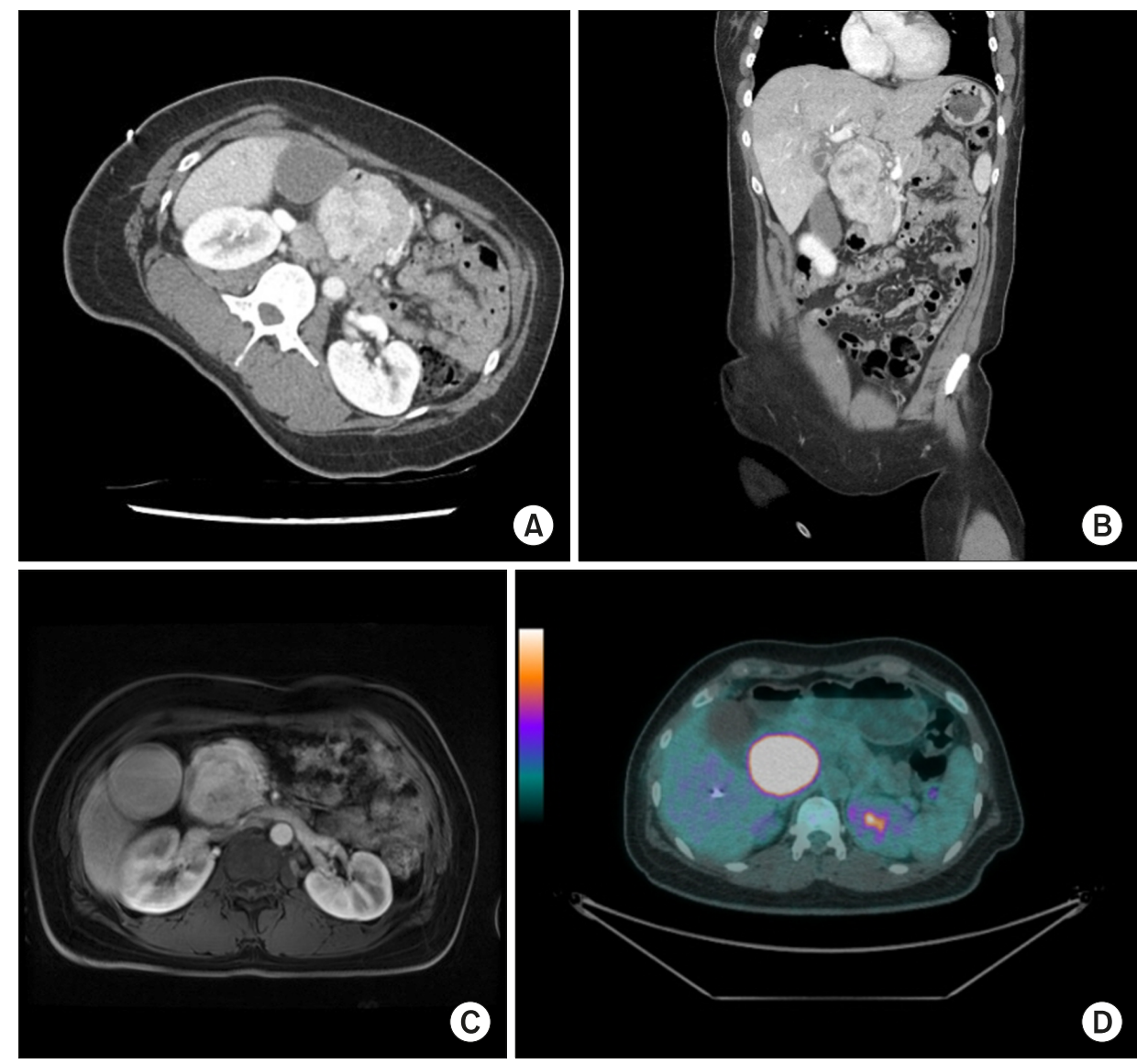

Fig. 4. Initial abdominal CT (A, B), MRI (C) and PET (D) images show $7.5 \mathrm{~cm}$ mass in the head of pancreas. 


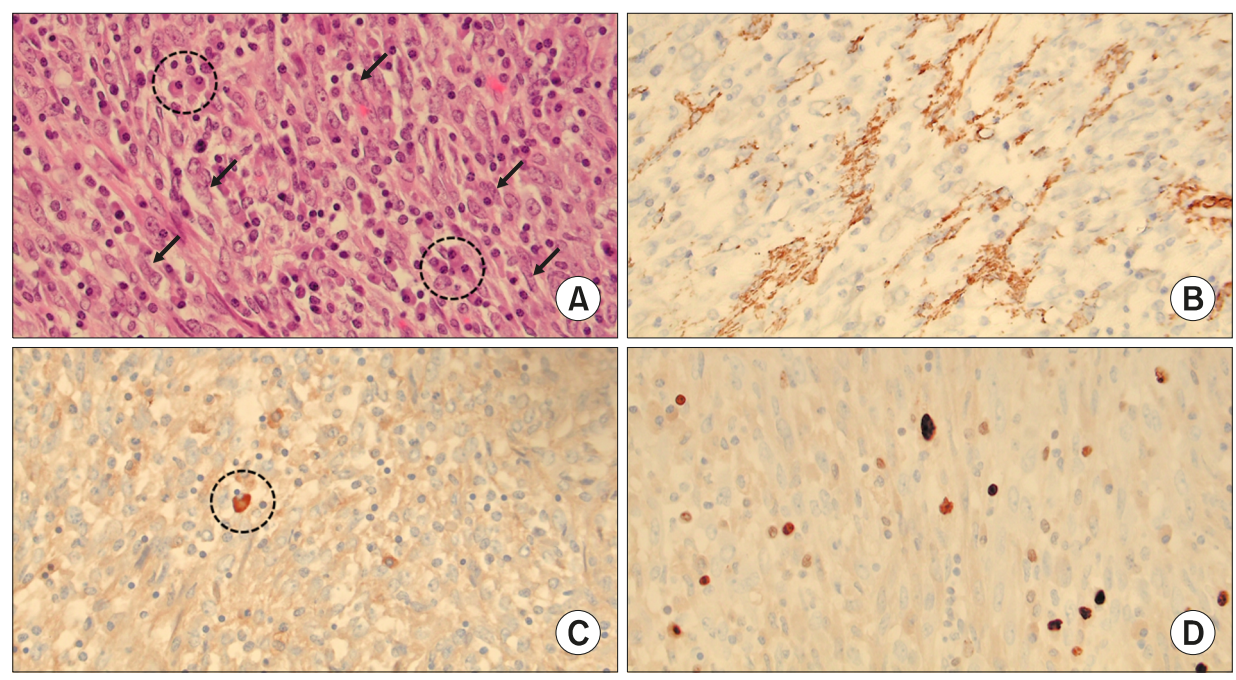

Fig. 5. (A) The tumor is composed of fascicles of plump spindle cells with oval nuclei and small nucleoli (arrows). Inflammatory cells, mostly plasma cells are seen either as small aggregates or more uniformly dispersed (dot-lined circle) $(\mathrm{H}$-E stain, $\times 400$ magnification). (B) The tumor cells show focal immunohistochemical positivity for smooth muscle actin (SMA, $\times 400$ magnification). (C) Immunohistochemical staining for IgG4, a marker of IgG4-positive plasma cell shows only one positive IgG4-positive cell per high power field (dot-lined circle) (IgG4, $\times 400$ magnification). (D) Ki-67 proliferation index is about $5 \%$ (Ki-67, ×400 magnification).

as small aggregates or were more uniformly dispersed.

The immunohistochemical staining test results were negative for ALK, S-100, CD23, CD34, and CD99, but the results were focally positive for only SMA (Fig. 5B). Only three IgG-positive plasma cells per high-power field were recognized (Fig. 5C), so IgG4-related pancreatitis was ruled out. The tumor showed low mitotic activity, and the Ki-67 proliferation index was about 5\% (Fig. 5D). As with the first case, no adjuvant treatment was provided to the patient after surgical excision of the tumor based on histological findings, and no evidence of recurrence was observed in the CT scan at the 6-month follow-up (Fig. 6). As far, about 1 year after surgery, the patient has been well without treatment and will be screened every 6 months.

\section{DISCUSSION}

Also known as inflammatory pseudotumors, IMTs refer to a heterogeneous group of lesions occurring in various organs that are histologically characterized by fibroblastic and myofibroblastic proliferation with inflammatory infiltrate. ${ }^{4}$ The current World Health Organization's (WHO's) classification for this rare tumor entity is a fibroblastic sarcoma or myofibroblastoma, which is a distinctive neoplasm of intermediate biological potential that may be ma-

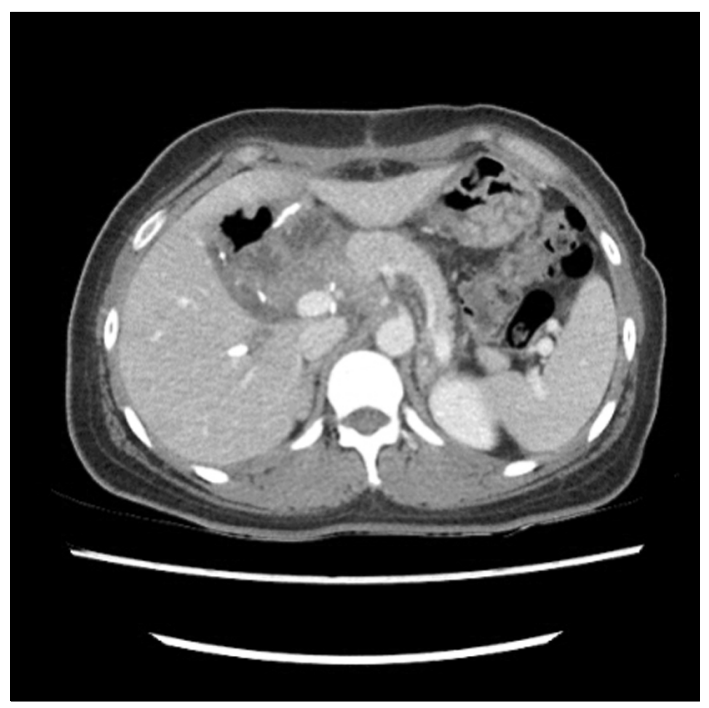

Fig. 6. After 6 month, no evidence of recurrence is observed in the $\mathrm{CT}$.

lignant or aggressive. ${ }^{3}$ We have reported 2 cases of large IMTs occurring in different locations: one in the retroperitoneum and the other in the pancreas head.

Generally, retroperitoneal tumors grow slowly and are usually presented as a solid abdominal palpable mass accompanied by fever, pain, or other symptoms. ${ }^{5}$ A differential diagnosis must be made to rule out benign fiber mesenteric tumors (such as mesenteric fibromatosis and sclerosing mesenteritis), lymphomas, soft tissue sarcomas, 
and metastatic malignancies. ${ }^{6}$ Because of its nonspecific symptoms and imaging findings, definitive diagnosis of IMT relies on histological evaluation. However, it is often difficult to obtain enough tissue using an ultrasonographyguided biopsy or a trans-endoscopic biopsy to reveal a definitive histological diagnosis. ${ }^{6}$ Additionally, although IMT may be detected during preoperative biopsy, surgical excision is mostly the treatment of choice for malignancy as there is no clear basis for conservative treatment. ${ }^{1,7-9}$

There have been recent reports of spontaneous regression of IMTs, ${ }^{10-12}$ which also indicated that surgical resection could be necessary for accurate diagnosis and treatment. The medical treatment is not clearly defined due to a lack of strong evidence in cases of large tumors, and palliative treatment cannot be recommended as a standard regimen. Moreover, in cases of a large IMTs, surgical treatment is required to relieve obstructive symptoms caused by the large mass and to exclude malignancy. This is why we performed complete surgical resection without pre-operative biopsy in both cases.

The prognosis of IMTs is favorable, and the 5-year survival rate is $91 \%{ }^{3}$ Although metastasis is a rare event in IMTs, ${ }^{13}$ recurrence rate is approximately $25 \%$ related to location, resectability and multinodularity. ${ }^{8}$ So far, neither of our cases have had recurrence or distant metastasis. However, long-term periodic follow-up could be necessary because of the possibility of local recurrence or distant metastasis of IMTs.

\section{CONCLUSION}

IMTs are a rare chronic inflammatory disease of unclear pathogenesis, and their pathological features are not those of a malignant tumor. They are very difficult to differentially diagnose from malignant diseases before complete surgical excision, especially in the pancreas. Therefore, according to the current literature and our experience, the primary choice for the exact diagnosis and proper treatment of IMTs is complete surgical resection.

\section{ETHICAL STATEMENT}

This study was approved by the ethics committee of Dong-A University Hospital (IRB No. DAUH IRB-20-090). The requirement for informed consent from patients was waived due to the retrospective nature of study.

\section{ACKNOWLEDGEMENTS}

This paper was not supported by any research fund.

\section{CONFLICT OF INTEREST}

The authors have no potential conflicts of interest to disclose.

\section{ORCID}

Eun Jeong Jang: https://orcid.org/0000-0002-8993-7769

Kwan Woo Kim: https://orcid.org/0000-0002-7432-6949

Sung Hwa Kang: https://orcid.org/0000-0002-5614-5968

Min Gyoung Pak: https://orcid.org/0000-0001-6142-4569

Song Hee Han: https://orcid.org/0000-0002-4564-7014

\section{AUTHOR CONTRIBUTIONS}

Conceptualization: KWK. Data curation: EJJ. Formal analysis: EJJ, KWK, SHK, MGP, SHH. Funding acquisition: None. Methodology: EJJ, KWK. Writing - original draft: EJJ, SHK, MGP. Writing - review \& editing: EJJ, KWK, SHH.

\section{REFERENCES}

1. Kovach SJ, Fischer AC, Katzman PJ, Salloum RM, Ettinghausen SE, Madeb R, et al. Inflammatory myofibroblastic tumors. J Surg Oncol 2006;94:385-391.

2. Thompson LDR. Inflammatory myofibroblastic tumor. Ear Nose Throat J 2019. doi: 10.1177/0145561319890165. [in press]

3. Ding $\mathrm{D}, \mathrm{Bu} \mathrm{X}$, Tian F. Inflammatory myofibroblastic tumor in the head of the pancreas with anorexia and vomiting in a 69year-old man: a case report. Oncol Lett 2016;12:1546-1550.

4. Battal M, Kartal K, Tuncel D, Bostanci O. Inflammatory myofibroblastic pancreas tumor: a case report. Clin Case Rep 2016;4: 1122-1124.

5. Tao YL, Wang ZJ, Han JG, Wei P. Inflammatory myofibroblastic tumor successfully treated with chemotherapy and nonsteroidals: a case report. World J Gastroenterol 2012;18:71007103.

6. Poves I, Alonso S, Jimeno M, Bessa X, Burdío F, Grande L. Retroperitoneal inflammatory pseudotumor presenting as a pancreatic mass. JOP 2012;13:308-311.

7. Zanchi C, Giurici N, Martelossi S, Cheli M, Sonzogni A, Alberti D. Myofibroblastic tumor of the pancreatic head: recurrent cholangitis. J Pediatr Gastroenterol Nutr 2015;61:e28-e29.

8. Panda D, Mukhopadhyay D, Datta C, Chattopadhyay BK, Chatterjee U, Shinde R. Inflammatory myofibroblastic tumor 
arising in the pancreatic head: a rare case report. Indian J Surg 2015;77:538-540.

9. Sim A, Lee MW, Nguyen GK. Inflammatory myofibroblastic tumour of the pancreas. Can J Surg 2008;51:E23-E24.

10. Matsubayashi H, Uesaka K, Sasaki K, Shimada S, Takada K, Ishiwatari $\mathrm{H}$, et al. A pancreatic inflammatory myofibroblastic tumor with spontaneous remission: a case report with a literature review. Diagnostics (Basel) 2019;9:150.

11. Yamaguchi J, Sakamoto Y, Sano T, Shimada K, Kosuge T.
Spontaneous regression of inflammatory pseudotumor of the liver: report of three cases. Surg Today 2007;37:525-529.

12. Zhao JJ, Ling JQ, Fang Y, Gao XD, Shu P, Shen KT, et al. Intra-abdominal inflammatory myofibroblastic tumor: spontaneous regression. World J Gastroenterol 2014;20:13625-13631.

13. Coffin CM, Hornick JL, Fletcher CD. Inflammatory myofibroblastic tumor: comparison of clinicopathologic, histologic, and immunohistochemical features including ALK expression in atypical and aggressive cases. Am J Surg Pathol 2007;31:509-520. 